\title{
Determinação do tempo de hidrodestilação e do horário de colheita no óleo essencial de menta
}

\author{
Ariana RMF de Oliveira ${ }^{1}$; Caroline N Jezler ${ }^{1}$; Rosilene A Oliveira ${ }^{2}$; Marcelo S Mielke ${ }^{1}$; Larissa CB Costa ${ }^{1}$

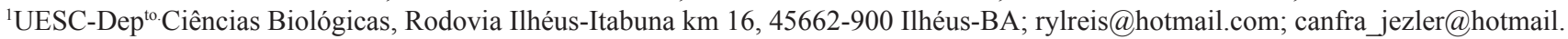

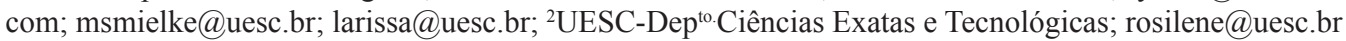

\section{RESUMO}

O tempo de hidrodestilação e o horário de colheita são informações importantes no estudo com plantas aromáticas, pois permitem maximizar o processo de extração e a quantidade de óleo essencial produzido. O objetivo do presente trabalho foi determinar o tempo de hidrodestilação e avaliar o efeito do horário de colheita no teor, rendimento e composição química do óleo essencial de Mentha $x$ piperita var citrata. Os tratamentos constituíram-se de quatro tempos de extração (30, 60, 90 e $120 \mathrm{~min})$ e cinco horários de colheita (9:00, 11:00, 13:00, 15:00 e 17:00 horas) com quatro repetições, dispostos em delineamento inteiramente casualizado. Após 60 minutos de hidrodestilação em aparelho de Clevenger ocorre estabilização do volume extraído. Em relação ao horário de colheita, ocorre uma variação significativa no teor de óleo essencial ao longo do dia, sendo o maior valor $(1,33 \%)$ encontrado na colheita realizada às 13:00h. Também foi verificada variação na composição química do óleo essencial, sendo que o $\alpha$-fenchol apresentou maior teor pela manhã e o cis-mirtanol pela tarde.

Palavras-chave: Mentha x piperita var citrata, planta medicinal, $\alpha$-fenchol, cis-mirtanol.

\begin{abstract}
Determination of hydrodistillation time and harvest moment on the essential oil of peppermint

The extraction time and harvest moment are important information in the study of herbs. They can maximize the efficiency of the extraction process and the amount of essential oil produced. The objective of this study was to determine the time of hydrodistillation and evaluate the effect of the harvest moment on the content, yield and chemical composition of essential oil of Menthax piperita var citrata. The treatments consisted of four extraction times (30, 60, 90 and 120 $\mathrm{min})$ and five harvest moments (9:00, 11:00, 13:00, 15:00 and 17:00 hours) with four replications arranged in a randomized design. After 60 minutes of hydrodistillation in Clevenger apparatus the volume extracted stabilized. In relation to the harvest moment, a significant variation was observed in the essential oil content throughout the day, the highest value $(1.33 \%)$ being found in samples taken at 13:00h. Variation in the chemical composition of essential oil was also noticed and higher contents of $\alpha$-fenchol and cis-myrtanol were verified in the morning and in the afternoon, respectively.
\end{abstract}

Keywords: Menthax piperita var citrata, medicinal plants, $\alpha$-fenchol, cis-myrtanol.

\section{(Recebido para publicação em 12 de agosto de 2010; aceito em 6 de fevereiro de 2012) (Received on August 12, 2010; accepted on February 6, 2012)}

\begin{abstract}
A família Lamiaceae tem grande importância econômica devido a sua abundância em espécies aromáticas (Costa, 2008) abrigadas em boa parte no gênero Mentha. A alevante (Mentha x piperita var citrata) é uma planta medicinal e aromática rica em óleo essencial, que é uma substância orgânica volátil encontrada em plantas de odor marcante (Almassy et al., 2007). A demanda por óleos essenciais derivados de plantas está em franca ascensão na utilização pela indústria farmacêutica, alimentícia, química e cosmética (Costa, 2008). Um grande desafio que envolve o uso de plantas medicinais e aromáticas é a obtenção de produtividades estáveis, em quantidade e qualidade desejadas, aumentando a confiabilidade da fitoterapia, uma vez que é imensamente variável a produção de princípios ativos nas regiões do país, justificando dessa forma
\end{abstract}

a importância dos estudos relacionados ao cultivo destas plantas, para suprir tais deficiências.

Alguns aspectos influenciam a produção de óleos essenciais, como os fatores genéticos e ambientais, e outros influenciam no rendimento, como o método e o tempo de extração. Na literatura consultada, o tempo de extração do óleo essencial para a espécie Mentha $x$ piperita apresentou grande variação (60 a $150 \mathrm{~min}$ ) (Souza et al., 2006; Valmorbida et al., 2006), sugerindo a importância de uma avaliação prévia do tempo de extração do óleo essencial para cada espécie, levando em consideração as particularidades de método e aparelho de extração utilizados, visando-se obter a otimização do processo.

O horário de colheita é um aspecto que está relacionado não só ao ambiente como também à fisiologia da planta. Pre- sume-se que há simultaneamente, dois padrões de resposta do metabolismo secundário aos estímulos ambientais: as variações climáticas sazonais, de maior dimensão, no entanto, mais lentas, e as flutuações climáticas diárias, modificações menores e mais rápidas (Blank et al., 2005). De acordo com Souza et al. (2006), o horário de colheita é um parâmetro relevante para a produção de óleo essencial, pois pode interferir no teor e na composição química das plantas ao longo do dia. A influência do horário de colheita no teor e na composição química de óleo essencial também tem sido investigada por alguns autores em outras espécies medicinais como: Ocimum basilicum (Carvalho-Filho et al., 2006), Andropogon sp. (Nascimento et al., 2006), Porophyllum ruderale (Fonseca et al., 2007), Melissa officinalis (Blank et al., 2007), 
Ocimum selloi e Rosmarinus officinalis (Gonçalves et al., 2009). Tais autores encontraram respostas individualizadas para cada espécie estudada, não havendo um padrão definido para a produção de óleos essenciais nas plantas aromáticas.

Em trabalhos realizados por Garlet (2007) com a espécie M. x piperita var. citrata o teor de óleo essencial variou de 0,76 a $1,60 \%$, obtendo um rendimento de 2,45 a 3,42 g/planta e produzindo como compostos majoritários o linalol $(44,97-53,47 \%)$ e acetato de linalila $(28,07$ - 33,86\%) quando cultivada em hidroponia.

Visando a otimização do rendimento e o estabelecimento de boas práticas de colheita para menta, o objetivo do trabalho foi determinar o tempo de hidrodestilação e avaliar o efeito do horário de colheita no teor, rendimento e composição química do óleo essencial de menta.

\section{MATERIAL E MÉTODOS}

O experimento foi conduzido no Horto de Plantas Medicinais da Universidade Estadual de Santa Cruz, localizada na cidade de Ilhéus (14\%47'48's; $39^{\circ} 10^{\prime} 26^{\prime \prime} \mathrm{W} ; 12 \mathrm{~m}$ de altitude), em dezembro de 2009.

As mudas de menta foram obtidas através do plantio de estacas apicais de $10 \mathrm{~cm}$, em bandejas de polietileno de 150 células utilizando areia e solo como substrato (1:2). Após 30 dias, as mudas foram transplantadas para os canteiros em espaçamento $30 \times 20 \mathrm{~cm}$ e colhidas aos 60 dias após o transplante. A irrigação e capina foram feitas de forma manual sempre que necessário. O material vegetal foi devidamente identificado e a exsicata encontra-se depositada no herbário da UESC, registrada pelo número 14086, com determinação feita por Dr. Ray Harley do Royal Botanic Gardens, Kew, Inglaterra.

Para determinar o tempo de máxima extração de óleo essencial utilizou-se o método de hidrodestilação em aparelho de Clevenger utilizando quatro repetições de $100 \mathrm{~g}$ de biomassa foliar fresca em balão de 3 L contendo 1,5 L de água destilada registrando-se o volume de óleo extraído a cada 30 minutos, pelo tempo necessário até atingir a estabilização. Determinado o tempo ideal, utilizou-se o mesmo procedimento de extração para o experimento de horário de colheita, porém foi utilizada biomassa foliar seca. O óleo essencial foi separado do hidrolato usando diclorometano, seco com sulfato de sódio anidro e concentrado. O teor foi determinado com base no volume extraído por 100 $\mathrm{g}$ de matéria vegetal $(\% \mathrm{p} / \mathrm{v})$.

As amostras dos óleos foram analisadas, no Laboratório de Fisiologia Vegetal da UESC, por cromatografia gasosa, utilizando o aparelho Varian Saturno 3800 equipado com detector de ionização de chama (FID), utilizando coluna capilar de sílica fundida $(30 \mathrm{~m}$ x 0,25 mm) com fase estacionária VF$-5 \mathrm{~ms}(0,25 \mu \mathrm{m}$ de espessura de filme), tendo hélio como gás arraste, fluxo de $1,2 \mathrm{~mL} \mathrm{~min}^{-1}$. As temperaturas do injetor e detector foram de 250 e $280^{\circ} \mathrm{C}$, respectivamente. A temperatura da coluna para as análises teve início a $50^{\circ} \mathrm{C}$, aumentando $3^{\circ} \mathrm{C}$ até $160^{\circ} \mathrm{C}$ seguido de aumento de $10^{\circ} \mathrm{C}$ até $220^{\circ} \mathrm{C}$. Foram injetados $1 \mu \mathrm{L}$ de solução a $10 \%$ de óleo em clorofórmio, com razões split 1:10. A concentração dos constituintes voláteis foi calculada através da área da integral de seus respectivos picos, relacionadas com a área total de todos os constituintes da amostra. As análises qualitativas dos óleos essenciais foram realizadas usando-se um espectrômetro de massas Varian Chromopack 2000 $\mathrm{MS} / \mathrm{MS}$, equipado com a mesma coluna capilar de sílica fundida. O modo de operação foi impacto eletrônico a $70 \mathrm{eV}$. As programações de temperatura da coluna foram as mesmas usadas nas análises de cromatografia a gás. Os diversos constituintes químicos dos óleos essenciais foram identificados através da comparação computadorizada com a biblioteca do aparelho, literatura e índice de retenção de Kovats (Adams, 1995). Os índices de retenção de Kovats (IK) foram calculados através da injeção de uma série de padrões de n-alcanos $\left(\mathrm{C}_{8}\right.$ $\mathrm{C}_{26}$ ), injetados nas mesmas condições cromatográficas das amostras.

Durante o dia do experimento de horário de colheita, das 7:30 às 17:00 horas a radiação fotossinteticamente ativa (RFA) foi monitorada por meio de um sensor de radiação luminosa S-LIA-M003, acoplado à uma estação climatológica Hobo Micro Station Data Logger (Onset, USA), enquanto a temperatura e a umidade relativa do ar foram registradas utilizando-se um sensor microprocessado Hobo H8 Pro Series (Onset, USA).

Para a análise da influência do horário de colheita no óleo essencial foi utilizado o delineamento inteiramente casualizado, testando cinco horários de colheitas (9:00, 11:00, 13:00, 15:00 e 17:00h), com quatro repetições. Os resultados foram analisados estatisticamente por meio da análise de variância e teste de médias (Teste de Tukey, 5\% de probabilidade).

\section{RESULTADOS E DISCUSSÃO}

O ensaio para determinação do tempo de máxima extração de óleo essencial de menta demonstrou que após 60 minutos de hidrodestilação em aparelho de Clevenger obteve-se um teor de $0,5 \mathrm{~mL}$ e ocorre estabilização do volume extraído (Tabela 1). Esse tempo foi inferior ao encontrado por Ehlert et al. (2006) para outras espécies medicinais: 130 minutos de extração para Cymbopogon citratus, 150 minutos para as espécies Cymbopogon

Tabela 1. Volume de óleo essencial de Mentha x piperita var. citrata em relação ao tempo de extração de $100 \mathrm{~g}$ de biomassa foliar fresca (essential oil volume of Mentha x piperita var citrata depending on the extraction time from $100 \mathrm{~g}$ of fresh leaf biomass). Ilhéus, UESC, 2009.

\begin{tabular}{lc}
\hline $\begin{array}{c}\text { Tempo de extração } \\
(\mathbf{m i n})\end{array}$ & $\begin{array}{c}\text { Volume de } \\
\text { óleo }(\mathbf{m L})\end{array}$ \\
\hline 30 & $0,3 \mathrm{~b}$ \\
60 & $0,5 \mathrm{a}$ \\
90 & $0,5 \mathrm{a}$ \\
120 & $0,5 \mathrm{a}$ \\
\hline${ }^{*} \mathrm{CV}(\%)$ & 14,14 \\
\hline
\end{tabular}

*Coeficiente de variação. Médias seguidas por letras iguais nas linhas não diferem entre si pelo Teste de Tukey ao nível de 5\% de probabilidade [coefficient of variation. Means followed by same letters in lines do not significantly differ by Tukey test $(\mathrm{p} \leq 0.05)]$. 

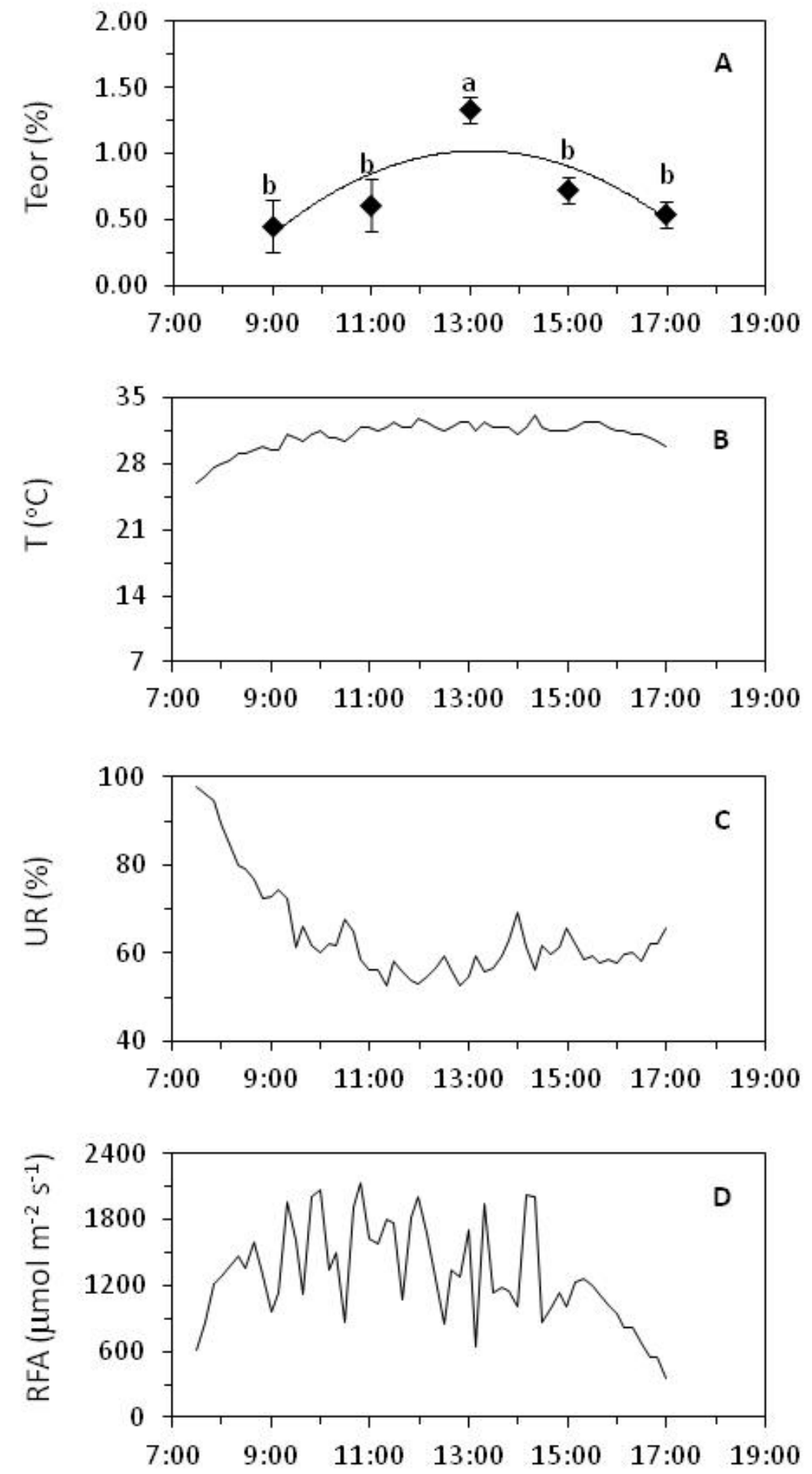

Hora

Figura 1. Teor de óleo essencial de Mentha x piperita var citrata (A), temperatura (B), umidade relativa do ar (C) e radiação fotossinteticamente ativa (D) ao longo do dia. Médias seguidas por letras distintas diferem entre si pelo teste de Tukey $(\mathrm{p} \leq 0,05)$. Barras correspondem ao desvio padrão da média, $\mathrm{n}=5$ [essential oil yield of Mentha $\mathrm{x}$ piperita var citrata (A), temperature (B), relative humidity (C) and photosyntethic active radiation (D) throughout the day. Means followed by distinct letters differ by Tukey test $(\mathrm{p} \leq 0.05)$. Bars represent the standard deviation, $\mathrm{n}=5$ ]. Ilhéus, UESC, 2009. winterianus, Aristolochia sp, Hyptis pectinata e Hyptis fruticosa, 160 minutos para Lippia sidoides e 230 minutos para Eucalyptus globulus. Essa diferença ocorre não apenas entre diferentes espécies, como também dentro da mesma espécie. Para Mentha $x$ piperita, foram encontrados diferentes tempos de extração: 60 minutos (Souza et al., 2006), 90 minutos (David \& Boaro, 2009), 120 minutos (Garlet, 2009) e 150 minutos de extração (Valmorbida et al., 2006).

Em relação ao horário de colheita verificou-se que houve variação significativa no teor de óleo essencial da menta ao longo do dia. Na colheita realizada às 13:00 $\mathrm{h}$ obteve-se o maior teor de óleo essencial $(1,33 \%)$ sendo que esse tratamento diferiu estatisticamente dos demais (Figura 1A).

Com relação aos dados climáticos, observou-se que a temperatura do ar foi menor no início da manhã, crescente ao longo do dia seguido de declínio após as 16:00 h (Figura 1B). A umidade relativa do ar foi máxima no início da manhã com 97,7\%, decrescendo até as 13:00 h depois do qual voltou a aumentar (Figura 1C). Já a radiação fotossinteticamente ativa (RFA), variou bastante ao longo do dia, sendo que no início da manhã e no final da tarde foram encontrados os menores valores (Figura 1D). Pode-se perceber que o período de maior produção de óleo essencial (13:00 h) coincidiu com os altos valores de temperatura do ar e RFA $\left(32,3^{\circ} \mathrm{C}\right.$ e 1711 $\mu \mathrm{mol} \mathrm{m} \mathrm{s}^{-2} \mathrm{~s}^{-1}$, respectivamente) e baixa umidade relativa do ar. Outros autores já associaram a produção diurna de óleos essenciais aos fatores ambientais como variações na temperatura e RFA, já que esses fatores podem influenciar diretamente o metabolismo primário afetando assim o metabolismo secundário e consequentemente a biossíntese de óleos essenciais (Santos et al., 2004; Marchese \& Figueira, 2005).

Estudos relacionados ao horário de colheita das plantas aromáticas são realizados e apresentam repostas diferenciadas conforme a espécie estudada. Pela manhã foram encontrados maiores teores de óleo essencial para: Andropogon sp. (Nascimento et al., 2006), Cymbopogon winterianus 
Tabela 2. Constituintes químicos do óleo essencial da biomassa seca de folhas de Mentha $x$ piperita var citrata em diferentes horários de colheita (chemical constituints of essential oil of dry biomass leaves of Mentha x piperita var citrata at different harvest moments). Ilhéus, UESC, 2009.

\begin{tabular}{lrrrrrr}
\hline & \multicolumn{5}{c}{ Horário de colheita } \\
\cline { 2 - 7 } Constituinte & \multirow{2}{*}{$\mathbf{I K}$} & $\mathbf{9 h}$ & $\mathbf{1 1 h}$ & $\mathbf{1 3 h}$ & $\mathbf{1 5 h}$ & $\mathbf{1 7 h}$ \\
\cline { 2 - 7 } & 856 & 0,32 & 0,36 & 0,94 & 0,40 & - \\
\hline tricicleno & 987 & - & 0,25 & 0,60 & - & - \\
$\alpha$-pineno & 1114 & 50,43 & 47,11 & 48,20 & 45,85 & 40,98 \\
$\alpha$-fenchol & 1183 & 0,87 & 0,99 & 0,75 & 0,76 & 0,67 \\
borneol & 1202 & 7,26 & 7,38 & 6,71 & 6,96 & 7,67 \\
$\alpha$-terpineol & 1230 & 1,36 & 1,44 & 1,24 & 1,42 & 1,64 \\
exo-acetato de fenchila & 1255 & 25,94 & 26,72 & 29,16 & 28,77 & 28,60 \\
cis-mirtanol & 1258 & 3,82 & 4,23 & 3,38 & 4,15 & 4,94 \\
trans-mirtanol & 1361 & 1,64 & 1,76 & 1,52 & 1,73 & 2,04 \\
acetato de citronila & 1423 & 0,97 & 1,08 & 0,84 & 1,12 & 1,13 \\
E-cariofileno & 1550 & 0,32 & 0,53 & 0,61 & 0,40 & 0,67 \\
valenceno & 1598 & 2,21 & 2,61 & 1,73 & 2,83 & 4,02 \\
guaiol & 1635 & 0,25 & 0,25 & - & 0,28 & 0,34 \\
$\beta$-eudesmol & & 0,32 & 0,61 & 1,54 & 0,40 & - \\
Monoterpenos & 91,32 & 89,63 & 90,96 & 89,64 & 86,54 \\
Monoterpenos oxigenados & & 1,25 & 1,61 & 1,45 & 1,52 & 1,80 \\
Sesquiterpenos & & 2,46 & 2,86 & 1,73 & 3,11 & 4,36 \\
Sesquiterpenos oxigenados & & 95,35 & 94,71 & 95,68 & 94,67 & 92,70 \\
Total identificado (\%) & & &
\end{tabular}

*IK= índice de Kovats experimental; - = Não detectado (IK= Kovats Index; - = Not detected).

(Blank et al., 2007) e Ocimum selloi (Gonçalves et al., 2009). Por outro lado, foi observado maior teor de óleo essencial no período da tarde para as espécies: Lippia alba (Santos et al., 2004), Cordia verbenacea (Souza et al., 2009) e Rosmarinus officinalis (Gonçalves et al., 2009). Esse fato evidencia a necessidade de estudos específicos, dificultando a definição de um comportamento padrão para a produção de óleos essenciais nas plantas aromáticas.

Em relação à composição química do óleo essencial é possível afirmar que houve variação na produção dos constituintes ao longo do dia (Tabela 2). Alguns autores também verificaram que houve influência do horário de colheita sob a composição química do óleo essencial em diferentes espécies aromáticas (Blank et al., 2005; Nascimento et al., 2006; Souza et al., 2006). Por outro lado, em Ocimum basilicum não foi verificada qualquer variação na composição química do óleo essencial durante o dia (Silva et al., 2003).

As análises permitiram a identificação de treze constituintes, perfazendo cerca de $94 \%$ da composição dos óleos essenciais. Esses óleos essenciais são ricos em monoterpenos oxigenados $(86,54$ a $91,32 \%)$. Os componentes majoritários foram o $\alpha$-fenchol $(40,98$ a $50,43 \%$ ), sendo observada a maior quantidade pela manhã, e o cis-mirtanol $(25,94$ a $29,16 \%)$ com a ocorrência da maior concentração pela tarde. Estes resultados diferem dos compostos majoritários, linalol e acetato de linalila, encontrados por Garlet (2007), o que permite pressupor que a espécie apresenta mais de um quimiotipo. Em menor quantidade foram identificados $\alpha$-terpineol (6,71 a 7,67\%), trans-mirtanol (3,38 a 4,94\%), guaiol $(1,73$ a $4,02 \%)$, exo-acetato de fenchila $(1,24$ a $1,64 \%)$ e acetato de citronila $(1,52$ a $2,04 \%$ ). Não foram detectados os compostos tricicleno às $17: 00 \mathrm{~h} ; \alpha$-pineno nos horários 9:00h, 15:00h e 17:00h e $\beta$-eudesmol às 13:00h (Tabela 2). A composição química dos óleos essenciais foi mais complexa no horário de 11:00 h, período em que a radiação fotossinteticamente ativa (RFA) está em ascensão. Uma maior produção de terpenos, principalmente os monoterpenos, no período de maior RFA, pode estar relacionada ao fato de que a biossíntese desses compostos é dependente da fotossíntese (Marchese \& Figueira, 2005) e de acordo com Taiz \& Zeiger (2004) os terpenos são biossintetizados a partir de metabólitos primários por duas rotas distintas, a rota do ácido mevalônico e a rota do metrileritriol fosfato.

Nas condições em que foi realizado o experimento é possível concluir para a espécie estudada que o tempo de máxima extração do óleo essencial em aparelho de Clevenger é de 60 minutos. Houve variação diurna no teor de óleo essencial, com o maior valor obtido às 13:00h, sendo que os constituintes majoritários $\alpha$-fenchol e cis-mirtanol também apresentaram variação no teor ao longo do dia.

\section{REFERÊNCIAS}

ADAMS RP. 1995. Identification of essential oil components by gas chromatography/mass spectrometry. Illinois Allured Publishing Corporation. $468 \mathrm{p}$.

ALMASSY A JUNIOR; ARMOND C; SILVA F; LOPES RC, CASALI, VWD. 2007. Curso sobre cultivo agroecológico de plantas medicinais. Cruz das Almas: Gráfica Nova Civilização. 96p.

BLANK AF; COSTA AG; ARRIGONI-BLANK MF; CAVALCANTE SCH; ALVES PB; INECCO R; EHLERT PAD; SOUZA IF. 2007. Influence of season, harvest time and drying on Java citronella (Cymbopogon winterianus Jowitt) volatile oil. Revista Brasileira de Farmacognosia 17: 557-564.

BLANK AF; FONTES SM; CARVALHO FILHO JLS; ALVES PB; SILVA-MANN R; MENDONÇA MC; ARRIGONI-BLANK MF; RODRIGUES MO. 2005. Influência do horário de colheita e secagem de folhas no óleo essencial de melissa (Melissa officinalis L.) cultivada em dois ambientes. Revista Brasileira de Plantas Medicinais 8: 73-78.

CARVALHO FILHO JLS; BLANK AF; ALVES PB; EHLERT PAD; MELO AS; CAVALCANTI SCH; ARRIGONI-BLANK MF; SILVA-MANN R. 2006. Influence of the harvesting time, temperature and drying period on basil (Ocimum basilicum L.) essencial oil. Revista Brasileira de Farmacognosia 16: $24-30$ 
COSTALCB. 2008. Condições culturais, anatomia foliar, processamento e armazenamento de Ocimum selloi em relação ao óleo essencial. Lavras: UFLA. 161p (Tese doutorado).

DAVID EFS; BOARO CSF. 2009. Translocação orgânica, produtividade e rendimento de óleo essencial de Mentha piperita L. cultivada em solução nutritiva com variação dos níveis de N, P, K e Mg. Revista Brasileira de Plantas Medicinais. 11: 236-246.

EHLERT PAD; BLANK AF; ARRIGONIBLANK MF; PAULA JWA; CAMPOS DA; ALVIANO CS. 2006. Tempo de hidrodestilação na extração de óleo essencial de sete espécies de plantas medicinais. Revista Brasileira de Plantas Medicinais 8: 79-80.

FONSECA MCM; CASALI VWD; BARBOSA LCA. 2007. Influência da época e do horário de colheita nos teores de óleo essencial e de taninos em couve-cravinho (Porophyllum ruderale) (Jacq.) Cassini. Revista Brasileira de Plantas Medicinais 9: 75-79.

GARLET TMB. 2007. Produtividade, teor e composição do óleo essencial de espécies de Mentha L. (Lamiaceae) cultivadas em hidroponia com variação de potássio. Santa Maria: UFSM. 112p. (Tese doutorado).

GONÇALVES GG; MANCINELLI RC; MORAES LAS. 2009. Influência do horário de corte no rendimento de óleo essencial de alfavaquinha e alecrim. Horticultura Brasileira 27: 108-112.

MARCHESE JA; FIGUEIRA GM. 2005. O uso de tecnologia pré e pós-colheita e boas práticas agrícolas na produção de plantas medicinais e aromáticas. Revista Brasileira de Plantas Medicinais 7: 86-96.

NASCIMENTO IB; INNECCO R; MATOS SH; BORGES NSS; MARCO CA. 2006. Influência do horário de corte na produção de óleo essencial de capim-santo (Andropogum sp). Revista Caatinga 19: 123-127.

SANTOS MRA; INNECCO R; SOARES AA. 2004. Caracterização anatômica das estruturas secretoras e produção de óleo essencial de Lippia alba (Mill) N. E. Br. em função do horário de colheita nas estações seca e chuvosa. Revista Ciência Agronômica 35:
37-383.

SILVA F; SANTOS RHS; DINIZ ER; BARBOSALCA; CASALI VWD; LIMA RR. 2003. Teor e composição do óleo essencial de manjericão (Ocimum basilicum L.) Revista Brasileira de Plantas Medicinais 6: 33-38.

SOUZA MF; NERY PS; MANGANOTTI SA; MATOS CC; MARTINS ER. 2009. Conteúdo de óleo essencial de Cordia verbenacea em diferentes horários de coleta. Revista Brasileira de Agroecologia 4: 2672-2675.

SOUZA WP; QUEIROGA CL; SARTORATTO A; HONÓRIO SL. 2006. Avaliação do teor e da composição química de óleo essencial de Mentha piperita (L.) Huds durante o período diurno em cultivo hidropônico. Revista Brasileira de Plantas Medicinais 8: 108-111.

TAIZ L; ZEIGER E. 2004. Fisiologia Vegetal. Porto Alegre: Artmed. 719 p. (3 ed.)

VALMORBIDA J; BOARO CSF; MARQUES MOM; FERRI AF. 2006. Rendimento e composição química de óleos essenciais de Mentha x piperita L. cultivada em solução nutritiva com diferentes concentrações de potássio. Revista Brasileira de Plantas Medicinais. 8: 56-61. 